\title{
Satisfacción laboral de los docentes de la Universidad Continental, 2014
}

\author{
Teachers' job satisfaction in the \\ Universidad Continental, 2014
}

\author{
Armando Carrillo Fernández \\ Universidad Continental \\ acarrillo@continental.edu.pe
}

\section{RESUMEN}

El objetivo de este trabajo fue describir la satisfacción laboral de los docentes de la Universidad Continental desde la perspectiva de los propios investigados a través de un enfoque cuantitativo. La investigación fue de carácter descriptivo, transversal. La técnica de recopilación de datos fue la encuesta, el instrumento fue un cuestionario que ha permitido extraer los significados de los sujetos estudiados y captar detalles y matices expresados en las escalas de respuesta. El instrumento fue validado por juicio de expertos, y se tomó como base la Escala General de Satisfacción Laboral. La muestra fue de 240 docentes que se encuentran en diversas condiciones laborales y cumplen funciones de carácter académico y administrativo. Algunos hallazgos son: respecto a la satisfacción laboral, de 240 encuestados el 92,9\% está satisfecho realizando la docencia, de 190 encuestados el 93,4 $\%$ está satisfecho realizando labores de docencia e investigación, de 122 encuestados el 78,7 \% siente satisfacción realizando docencia y deberes administrativos, de 121 encuestados el 94,3\% siente satisfacción realizando docencia y desempeñándose a la vez como coordinador de una asignatura, finalmente de 22 encuestados se tiene que el $68,2 \%$ siente satisfacción desempeñándose en la docencia y como director de una Escuela Académico Profesional. En conclusión, el 93,4 \% de los docentes de la Universidad Continental presentan un alto nivel de satisfacción laboral. Los docentes que son motivados en forma extrínseca, han demostrado un mejor desempeño.

Palabras clave: Satisfacción laboral, docencia, docentes.

\begin{abstract}
The purpose of this study was to describe the job satisfaction of teachers at the Continental University from their own perspective through a quantitative approach. The research was of descriptive and transversal type. The data collection technique was the survey; the instrument was a questionnaire which allowed extracting the information, captured details and nuances expressed in response scales. The instrument was validated by specialist criterion, and was based on the Job Satisfaction General Scale. The sample consisted of 240 teachers who had different working conditions and performed administrative and academic functions. Some findings regarding job satisfaction are, of the 240 respondents, $92.9 \%$ are satisfied doing the teaching, of 190 respondents, 93.4\% are satisfied doing teaching and research work, of 122 respondents, $78.7 \%$ feel satisfaction teaching and performing administrative duties, of 121 respondents, $94.3 \%$ feel satisfaction doing teaching and performing at the same time as a course coordinator, finally of 22 respondents, $68.2 \%$ feel satisfaction in teaching and performing as Directors of a Professional Academic School. In conclusion, 93.4\% of the Continental University teachers showed a high level of job satisfaction. In addition, teachers who are extrinsically motivated have shown a better performance.
\end{abstract}

Keywords: Job satisfaction, teaching, teachers.

Historial del artículo:

Recibido: 9 de enero de 2015. Aprobado: 21 de abril 2015. Disponible en línea: 30 de junio de 2015

1 Docente y director de la Carrera Profesional de Odontología de la Universidad Continental. 


\section{INTRODUCCIÓN}

El tema del trabajo cobra importancia ya que las personas pasan mucho tiempo de su vida en el mismo y es parte de su realización personal, cuando a las personas les gusta lo que hacen en su trabajo, lejos de seguir la máxima expresada por Xirau (1) "trabajar como un castigo y porque no hay más remedio" éste puede ser una fuente de satisfacciones, de felicidad y además remunerada.

La ausencia de satisfacción laboral a largo plazo provoca más gastos a la organización, tales como baja en productividad, absentismo, constantes errores, violación a normas de seguridad, baja en rendimiento, rotación de personal entre otros.

Esta investigación está dirigida a describir la satisfacción laboral de los docentes de la Universidad las experiencias negativas llevan a la insatisfacción personal y profesional que con frecuencia ocasionan el desgaste e incluso el rechazo de la profesión y llegan a afectar a la felicidad y bienestar del docente, al compromiso con la docencia y con el Centro Educativo.

Por su parte (4) Jaén explica en sus conclusiones, que los individuos que obtuviesen puntuaciones más altas en motivación, tendrían un mejor rendimiento general en el trabajo.

Alicia (5) llegó a las siguientes conclusiones, que los docentes presentan satisfacción laboral con relación a su capacidad pedagógica para realizar las tareas, ya que tienen dominio efectivo sobre los contenidos que imparten, conocimiento sobre planificación del proceso educativo y crean un ambiente favorable en el estudiante. Al analizar la dimensión desempeño laboral, se concluye que los docentes no están

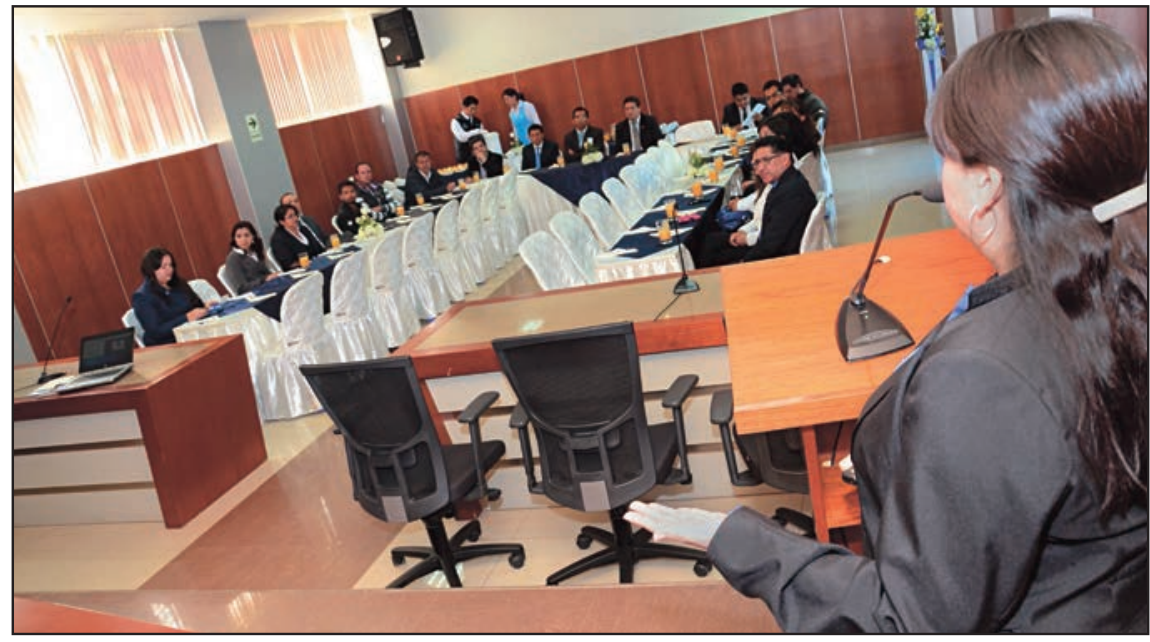

Figura $\mathrm{N}^{\circ}$ 1: Docentes de la Universidad Continental.

Continental en la ciudad de Huancayo en el año 2014 (figura $\mathrm{N}^{\circ} 1$ ).

El estudio realizado de Cámara (2), menciona que el compromiso organizacional de los profesores aumenta con el aumento de su antigüedad en el establecimiento educativo. Los resultados expresan que cuando los profesores presentan tiempo de trabajo reducido y consecuentemente un vínculo profesional menos sólido, presentan también niveles más bajos de conflicto.

Por su parte la investigación de Subaldo (3), indica que los resultados refuerzan el supuesto de partida que las experiencias positivas de los profesores en el ejercicio de la docencia producen satisfacción y conducen al desarrollo y a la realización personal y profesional, que efectivamente influyen en la calidad de la enseñanza y los aprendizajes de los alumnos. Por otra parte, satisfechos con las condiciones de trabajo, ya que el sueldo que percibe no está de acorde con las tareas que realizan, además, la institución no reconoce la labor que ejecutan al lograr satisfactoriamente sus objetivos.

López (6) en sus conclusiones explica que la muestra presenta máxima satisfacción al referirse a las relaciones personales con sus superiores y menor en relación al trato de igualdad y justicia que recibe en su empresa.

Navarro (7), menciona que entre los factores del entorno laboral en su mayor parte, la insatisfacción es debida a la baja cuantía de sus ingresos económicos, a su inestabilidad o a que el sistema de remuneración no está ligado al rendimiento de su trabajo.

Para Maldonado (8) en su investigación desde la 
perspectiva del factor docencia en el trabajo del profesor universitario, nos explica que la actividad de la enseñanza es el factor más valorado en el profesorado. Más que la satisfacción en investigación y en dirección y administración, disfrutan mucho los cursos que imparten, aunque algunos maestros se quejan del tamaño de los grupos y de la carga de trabajo.

Desde la perspectiva de satisfacción por edad del profesor; cuando la edad es considerada por sí sola, la calificación de la satisfacción laboral general de los maestros jóvenes es menor, y se incrementa progresivamente con la edad de los maestros universitarios.

A nivel nacional, Ventura (9) concluye que las percepciones de satisfacción laboral de los docentes directivos se manifiestan inicialmente de forma intrínseca estimuladas por el puesto en cuanto asumen el cargo. Estas percepciones, tienen que ver con las oportunidades que tienen para asumir retos y aprender competencias de gestión directiva.

La Universidad Continental a nivel de la presidencia de su directorio propone mejoras continuas que elevan su rendimiento como Institución Educativa, pero escazas veces se preguntan $\dot{i}$ Con qué tipo de motivación(es) está asociado la satisfacción del profesorado?, ¿̇cuál(es) son los principales factores de satisfacción en el trabajo de un profesor universitario?, ¿̇ué factores determinan el nivel de satisfacción en profesores universitarios?, ¿Cómo percibe el profesor universitario el reconocimiento interno por parte de la organización?

Es en este contexto es que surge el interés por identificar, cual es la satisfacción laboral de los docentes de la Universidad Continental con el fin de poder implementar mejoras en la gestión de recursos humanos, permitiendo tener un sistema integrado entre el empleador y el empleado bajo estándares básicos de satisfacción laboral.

El problema planteado ha sido ¿̇Cuál es el estado de satisfacción laboral de los docentes de la Universidad Continental de Huancayo en el año 2014?; el objetivo general fue, describir el estado de satisfacción laboral de los docentes de la Universidad Continental. La importancia de la investigación se expresa en que las personas pasan mucho tiempo de su vida en el trabajo y es parte de su realización personal, cuando a las personas les gusta lo que hacen en su trabajo, lejos de seguir la máxima "trabajar como un castigo y porque no hay más remedio" éste puede ser una fuente de satisfacciones, de felicidad y además remunerada. Fromm (10) expresaba en "La Revolución de la Esperanza", además está la necesidad de que cuando se haga, se realice con gusto, con satisfacción, con entusiasmo". De acuerdo a estos planteamientos, la ausencia de satisfacción laboral a largo plazo provoca más gastos a la organización, tales como baja en productividad, absentismo, constantes errores, violación a normas de seguridad, baja en el rendimiento, rotación de personal entre otros.

\section{MATERIAL Y MÉTODOS}

Se utilizó el diseño transversal descriptivo. Como técnica se aplicó la encuesta, porque es aplicado a nivel presencial y a distancia, es la técnica más conocida por su amplia difusión y alcance. El instrumento fue validado por juicio de expertos, y se tomó como base la Escala General de Satisfacción Laboral (11).

\section{RESULTADOS}

Algunos hallazgos son: respecto a la satisfacción laboral, de 240 encuestados el 92,9\% está satisfecho realizando la docencia, de 190 encuestados el 93,4 \% está satisfecho realizando labores de docencia e investigación, de 122 encuestados el 78,7 \% siente satisfacción realizando docencia y deberes administrativos, de 121 encuestados el 94,3\% siente satisfacción realizando docencia y desempeñándose a la vez como coordinador de una asignatura, finalmente de 22 encuestados se tiene que el 68,2 \% siente satisfacción desempeñándose en la docencia y como director de una Escuela Académico Profesional.

Tabla $N^{\circ}$ 1: Escala general de satisfacción laboral.

\begin{tabular}{lcrc}
\hline & Frecuencia & Porcentaje & $\begin{array}{c}\text { Porcentaje } \\
\text { acumulado }\end{array}$ \\
\hline Muy insatisfecho & 2 & $0,8 \%$ & $0,8 \%$ \\
Moderadamente insatisfecho & 5 & $2,1 \%$ & $2,9 \%$ \\
Ni satisfecho/insatisfecho & 9 & $3,8 \%$ & $6,7 \%$ \\
moderadamente satisfecho & 36 & $15,0 \%$ & $21,7 \%$ \\
Satisfecho & 111 & $46,3 \%$ & $67,9 \%$ \\
Muy satisfecho & 77 & $32,1 \%$ & $100,0 \%$ \\
Total & 240 & $100,0 \%$ & \\
\hline
\end{tabular}


Tabla N²: Estado de la satisfacción laboral de los docentes en el campos de la docencia.

\begin{tabular}{|c|c|c|c|}
\hline & Frecuencia & Porcentaje & $\begin{array}{l}\text { Porcentaje } \\
\text { acumulado }\end{array}$ \\
\hline Muy insatisfecho & 1 & $0,4 \%$ & $0,4 \%$ \\
\hline Insatisfecho & 1 & $0,4 \%$ & $0,8 \%$ \\
\hline Moderadamente insatisfecho & 5 & $2,1 \%$ & $2,9 \%$ \\
\hline Ni satisfecho ni insatisfecho & 10 & $4,2 \%$ & $7,1 \%$ \\
\hline Moderadamente satisfecho & 31 & $12,9 \%$ & $20,0 \%$ \\
\hline Satisfecho & 103 & $42,9 \%$ & $62,9 \%$ \\
\hline Muy satisfecho & 89 & $37,1 \%$ & $100,0 \%$ \\
\hline Total & 240 & $100,0 \%$ & \\
\hline
\end{tabular}

Tabla N 3: Estado de la satisfacción laboral de los docentes en los campos de la docencia e investigación.

\begin{tabular}{|c|c|c|c|}
\hline & Frecuencia & Porcentaje & $\begin{array}{l}\text { Porcentaje } \\
\text { acumulado }\end{array}$ \\
\hline Moderadamente insatisfecho & 1 & $0,5 \%$ & $0,5 \%$ \\
\hline Ni satisfecho/insatisfecho & 10 & $5,3 \%$ & $5,8 \%$ \\
\hline Moderadamente satisfecho & 29 & $15,3 \%$ & $21,1 \%$ \\
\hline Satisfecho & 94 & $49,5 \%$ & $70,5 \%$ \\
\hline Muy satisfecho & 56 & $29,5 \%$ & $100,0 \%$ \\
\hline Total & 190 & $100,0 \%$ & \\
\hline
\end{tabular}

Tabla $N^{\circ}$ 4: Estado de la satisfacción laboral de los docentes en los campos de la docencia y deberes administrativos.

\begin{tabular}{lcrrr}
\hline & Frecuencia & Porcentaje & $\begin{array}{r}\text { Porcentaje } \\
\text { acumulado }\end{array}$ & $4,1 \%$ \\
\hline Moderadamente insatisfecho & 5 & $4,1 \%$ & $21,3 \%$ \\
Ni satisfecho/insatisfecho & 21 & $17,2 \%$ & $81,1 \%$ \\
Moderadamente satisfecho & 73 & $59,8 \%$ & $100,0 \%$ \\
Satisfecho & 23 & $18,9 \%$ & $100,0 \%$ \\
Total & 122 & & \\
\hline
\end{tabular}

Tabla $N^{\circ}$ 5: Estado de la satisfacción laboral de los docentes en los campos de la docencia y coordinador de curso.

\begin{tabular}{|c|c|c|c|}
\hline & Frecuencia & Porcentaje & $\begin{array}{l}\text { Porcentaje } \\
\text { acumulado }\end{array}$ \\
\hline Moderadamente insatisfecho & 1 & $0,8 \%$ & $0,8 \%$ \\
\hline Ni satisfecho/insatisfecho & 6 & $5,0 \%$ & $5,8 \%$ \\
\hline Moderadamente satisfecho & 14 & $11,6 \%$ & $17,4 \%$ \\
\hline Satisfecho & 64 & $52,9 \%$ & $70,2 \%$ \\
\hline Muy satisfecho & 36 & $29,8 \%$ & $100,0 \%$ \\
\hline Total & 121 & $100,0 \%$ & \\
\hline
\end{tabular}

\section{DISCUSIÓN}

La Guía Universidad César Vallejo (12) detalla que la calidad de la educación ha dejado de entenderse como una consecuencia natural de la educación y se convierte en una de las principales prioridades del sistema educativo, la evaluación de la calidad de las instituciones educativas son cada vez más relevantes como una de las formas para elevar la calidad de éstas dentro del nuevo enfoque de gestión, asimismo el compromiso de los trabajadores, el movimiento de cero defectos que introduce el enfoque de aseguramiento de la calidad y la atención a los recursos humanos. Esta propuesta afirma que si la dirección general exige un trabajo perfecto, el camino para obtenerlo es motivando a los trabajadores y monitorizando los procesos, es en este nivel donde la Universidad Continental encontrará las respuestas a las mejoras que propone para mejorar su desempeño.

Contrariamente a Subaldo (3), existe una percepción de parte del colaborador que se tiene ausencia de 
satisfacción laboral en nuestra universidad, lo que a largo plazo provoca más gastos a la organización, tales como baja en productividad, absentismo, constantes errores, violación a normas de seguridad, baja en rendimiento, rotación de personal entre otros.

En concordancia relativa con a Jaén (4), los docentes que son motivados en nuestra universidad de forma extrínseca, han demostrado mejor rendimiento académico. Antagónicamente a las conclusiones de Alicia (5), en la Universidad Continental los docentes no se sienten satisfechos al tener un patrón rígido para la elaboración de las sus clases. Pero si coincide con Alicia (5) al analizar la dimensión de desempeño laboral, los docentes no están satisfechos con las condiciones de trabajo, ya que el sueldo que perciben no está de acorde con las tareas que realizan, además, la institución no reconoce la labor que ejecutan al lograr satisfactoriamente sus objetivos.

Las conclusiones de López (6), no parecen reflejarse en la Universidad Continental, dada una escasa relación de los docentes con sus superiores. La conclusión de Navarro (7) también es un factor común en nuestra universidad. Respecto a Maldonado (8) existen coincidencias en la actividad de la enseñanza considerando que es el factor más valorado en el profesorado. Mientras que vemos una mínima discrepancia con Ventura (9) que observa los factores extrínsecos como intrínsecos como distribuidos sin una predominancia de uno de ellos.

Finalmente consideramos que esta investigación es un aporte para mejorar el plan de trabajo de las oficinas involucradas en la gestión humana. Un cambio en la gestión de la calidad propuesta por la universidad desde la perspectiva de los recursos humanos permitirá a futuro mejorar los procesos inherentes al trabajo en la universidad. Herzberg (13), habla de la importancia de la atención al trabajador, Maslow (14), explicó sobre las empresas que promueven la actitud creativa en el trabajo obtienen personas con actitud creativa y son capaces de hacer frente a los cambios rápidos e inevitables. Elton (15) descubrió que los aspectos humanos (entre ellos el sentimiento de pertenecer a un grupo de trabajo cohesivo y agradable) despiertan sentimientos de afiliación, competencia y logro, necesidades que por mucho tiempo han sido insatisfechas en el trabajo.

\section{REFERENCIAS BIBLIOGRÁFICAS}

1. Xirau R. Introducción a la historia de la Filosofía. México D.F.: Universidad Nacional Autónoma de México; 1987.

2. Câmara SM. Conflicto, Cultura y Compromiso
Organizacional: Un estudio en profesorado de las instituciones educativas de la Región Autónoma de Madeira. [Tesis doctoral]. Cádiz: Universidad de Cádiz; 2012.

3. Subaldo L. Las repercusiones del desempeño docente en la satisfacción y el desgaste del profesorado. [Tesis doctoral]. Alcalá de Henares: Universidad de Alcalá; 2012.

4. Jaén M. Predicciones del rendimiento laboral a partir de indicadores de motivación, personalidad y percepción de factores psicosociales. Madrid: Universidad Complutense; 2010.

5. Alicia C. Satisfacción laboral de los docentes del decanato de Administración y Contaduría de la UCLA con relación al clima institucional, bajo un enfoque prospectivo. [Tesis doctoral]. Barquisimeto: Universidad Centroccidental "Lisandro Alvarado"; 2010.

6. López M. Consecuencias psicosociales del trabajo en personal de enfermería como indicadores subjetivos de rendimiento desde el enfoque de la gestión de recursos humanos. [Tesis doctoral]. Murcia: Universidad de Murcia; 2009.

7. Navarro E. Aportación al estudio de la satisfacción laboral de los profesionales técnicos del sector de la construcción: una aplicación cualitativa en la Comunidad Valenciana. [Tesis doctoral]. Valencia: Universidad Politècnica de València; 2008.

8. Maldonado V. La satisfacción en el trabajo y su relación con la efectividad ética organizacional. Caso específico del profesorado universitario. [Tesis doctoral]. San Sebastián: Universidad de Deusto Campus San Sebastián; 2007.

9. Repositorio Digital de tesis PUCP [Internet]. Lima: PUCP; [04 de abril de 2014]. Satisfacción laboral en docentes-directivos con sección a cargo de cuatro instituciones de educación inicial pertenecientes a la UGEL 07 [ 1 pantalla]. Disponible en: http://tesis.pucp.edu.pe/ repositorio/handle/123456789/4734

10. Fromm E. La Revolución de la Esperanza. Austin: Fondo de cultura económica; 2009.

11. Locke E. The relationship of task success to task liking and satisfaction. J Appl Psychol. 1965; 49(5): 379-85.

12. Facultad de Educación de la Universidad César Vallejo. Cultura innovadora y productiva. Trujillo: UCV; 2010.

13. Herzberg F, Mausner B, Peterson RD, Capwell DF. Job attitudes: Review of research and opinion. Pittsburgh: Psychological Service of Pittsburgh; 1957.

14. Maslow A. El hombre autorrealizado. 119 $\mathrm{ed}$. Barcelona: Kairos; 1995.

15. Elton M. El experimento de Hawthorne en la Western Electric Company. En: España. Ministerio para las Administraciones Públicas. Teoría de la organización, Vol.1. Madrid: INAP; 1993. 